\title{
8. Discusión multidisciplinaria para el diagnóstico de la fibrosis pulmonar idiopática
}

\author{
MATÍAS FLORENZANO V.*, FELIPE REYES C.** y ÁLVARO UNDURRAGA P.***
}

\section{Multidisciplinary discussion for the diagnosis of idiopathic pulmonary fibrosis}

Prior to the publication of the 2002 ATS / ERS classification of idiopathic interstitial pneumonias (IIP), the histopathological evaluation was considered the gold standard for the diagnosis of interstitial lung diseases (ILD). However, several subsequent studies showed that interobserver concordances between expert lung pathologists were surprisingly poor, since histopathological appearances may overlap between different entities. Therefore, a new diagnostic system that serves as a gold standard in patients with ILD became necessary. This is how the concept of multidisciplinary discussion was born, to refer to a meeting that allows the integration of all the clinical, radiological and pathological data available for an individual patient and thus be able to determine a working diagnosis.

Key words: Idiopathic Pulmonary Fibrosis; Pathologists, Idiopathic Interstitial Pneumonias; Lung Diseases, Interstitial.

\section{Resumen}

Antes de la publicación de la clasificación ATS/ERS 2002 de las neumonías intersticiales idiopáticas (NII), la evaluación histopatológica se consideraba la referencia de oro para el diagnóstico de las enfermedades pulmonares intersticiales (EPI). Sin embargo, varios estudios posteriores mostraron que las concordancias interobservador entre anatomopatólogos expertos torácicos eran sorprendentemente pobres ya que las apariencias histopatológicas pueden superponerse entre entidades distintas. Por lo anterior, se hace necesario un nuevo sistema diagnóstico que sirva de patrón de oro en pacientes con EPI. Es así como nace el concepto de discusión multidisciplinaria, para referirse a una reunión que permita la integración de todos los datos clínicos, radiológicos y patológicos disponibles para un paciente individual y así poder determinar un diagnóstico de trabajo.

Palabras clave: Fibrosis pulmonar idiopática; Patólogos; Neumonías Intersticiales Idiopáticas; Enfermedades Pulmonares; Intersticiales.

Antes de la publicación de la clasificación 2002 ATS/ERS de las neumonías intersticiales idiopáticas (NII), la evaluación histopatológica se consideraba la referencia de oro para el diagnóstico de las enfermedades pulmonares intersticiales (EPI $)^{1}$. Sin embargo, varios estudios posteriores mostraron que las concordancias inter-observador entre anatomopatólogos expertos torácicos eran sorprendentemente pobres ${ }^{2}$ ya que las aparien- cias histopatológicas pueden superponerse entre entidades distintas. Flaherty y cols. ${ }^{3}$ demostraron que el $26 \%$ de los pacientes tenían diferentes diagnósticos histopatológicos asignados a diferentes lóbulos y que la presencia de un patrón neumonía intersticial usual (NIU) en cualquier muestra confería un mal pronóstico. En términos prácticos un diagnóstico clínico basado exclusivamente en la histología puede ser inexacto. Del

\footnotetext{
* Instituto Nacional del Tórax y Clínica Las Condes. Santiago, Chile.

** Coordinador Comisión de Enfermedades pulmonares intersticiales difusas, SER Chile. Hospital Clínico Universidad de Chile, Instructor Universidad de Chile. Santiago, Chile.

*** Profesor Adjunto Universidad de Chile. Instituto Nacional del Tórax y Clínica Las Condes. Santiago, Chile.
} 
mismo modo, la concordancia interobservador entre los patrones radiológicos de la tomografía computada (TC) de tórax es moderada a alta sólo para el patrón de NIU ${ }^{4}$; los otros patrones distan mucho de esta buena correlación.

Por lo anterior, se hace necesario un nuevo sistema diagnóstico que sirva de patrón de oro en pacientes con EPI. Es así como nace el concepto de discusión multidisciplinaria, para referirse a una reunión que permita la integración de todos los datos clínicos, radiológicos y patológicos disponibles para un paciente individual y así poder determinar un diagnóstico de trabajo. El objetivo principal del comité multidisciplinario (CMD) es discutir y establecer el diagnóstico y las estrategias de los tratamiento adecuados, por ejemplo, la prescripción de terapia antifibrótica en pacientes con fibrosis pulmonar idiopática (FPI) u otras enfermedades pulmonares fibrosantes progresivas.

\section{¿Quiénes deben participar en el Comité multidisciplinario?}

La respuesta no está absolutamente zanjada: neumólogos, radiólogos y patólogos son importantes en el proceso diagnóstico. En nuestra opinión es de vital importancia la presencia de un reumatólogo. En un estudio reciente se informa que la inclusión del reumatólogo en el CMD en la evaluación de una EPI de reciente diagnóstico permitió la reclasificación del $21 \%$ de los pacientes ${ }^{5}$. Los diagnósticos de vasculitis ANCA, síndrome antisintetasa e IPAF (interstitial pneumonia with autoimmune features) fueron los diagnósticos más frecuentes realizados posterior a la evaluación por reumatología. La inclusión inicial del reumatólogo en el CMD, podría evitar la realización de procedimientos diagnósticos adicionales como la biopsia pulmonar.

Para que el CMD cumpla efectivamente su papel debería formarse en sitios donde existan los especialistas con experiencia en el manejo de enfermedades pulmonares difusas y tengan un número de casos que aseguren mantener y aumentar dicha experiencia.

La participación de un CMD en la evaluación de pacientes EPI ha mostrado altos niveles de confianza en el diagnóstico de FPI y EPI asociadas a enfermedades del tejido conectivo (ETC). Esto es menor en otras entidades como neumonitis por hipersensibilidad (NHS) o de neumonías intersticiales no específicas (NINE) idiopáticas, a pesar de lo cual la contribución del CMD es mayor que la opinión por separado del grupo de expertos (es decir, neumólogo, radiólogos, patólogos o reumatólogo) de forma aislada ${ }^{6}$.

\section{¿Todos los casos con EPI deben ser discutidos en Comité multidisciplinario? ${ }^{7}$}

La respuesta no es absoluta y dependerá de la experticia del grupo. Podríamos recomendar que los grupos menos experimentados sesionaran de forma periódica pues la discusión enriquece el conocimiento y el ejercicio docente, sin embargo, en la realidad nacional es poco probable llevar esto acabo. Nosotros recomendamos que, en la medida de lo posible, se presenten en discusión todos aquellos casos que generen dudas sobre el diagnóstico, la necesidad de estudios complementarios o una decisión terapéutica que agreguen mayor costo tanto de recursos como riesgos para el paciente.

Un simple ejemplo resaltará este punto: el patrón radiológico definitivo de NIU en la tomografía computada (TC) de tórax de alta resolución es altamente predictor de patrón NIU histológico, lo que permite obviar una biopsia quirúrgica. Por lo tanto, si no hay causas secundarias identificadas (drogas, exposiciones, enfermedades del tejido conectivo) un patrón típico de NIU en TC de alta resolución es diagnóstico de $\mathrm{FPI}^{8}$. Un patrón "NIU probable" (igual al definitivo, pero sin panal de abejas), también es sugerente de un patrón histológico de NIU, sobre todo, en adultos mayores con bronquiectasias por tracción profusas ${ }^{9}$. Sin embargo, la presencia de un patrón indeterminado o elementos alternativos para NIU deben levantar sospecha de otra enfermedad pulmonar intersticial como neumonitis por hipersensibilidad, NINE, sarcoidosis u otra ${ }^{10}$. La discusión en CMD de estos casos se hace fundamental.

La propuesta del último consenso internacional $^{11}$ es plantear la reunión del CMD en dos momentos del proceso: para definir necesidad de fibrobroncoscopía y/o biopsia quirúrgica y luego para definir diagnóstico final de trabajo con la información de la histología (Figura 1).

En estos casos el CMD puede proponer, estudios complementarios adicionales. La fibrobroncoscopía (FBC) para lavado bronquioloalveolar (LBA) y biopsias transbronquiales (BTB). El LBA es particularmente útil al demostrar linfocitosis ante diagnóstico diferencial de NHS y sarcoidosis o eosinofilia en neumonía eosinofílica. En general, en aquellos patrones radiológicos predominantemente periféricos se prefiere la biopsia quirúrgica por videotoracoscopía. En aquellas enfermedades intersticiales peribroncovasculares o difusas se puede empezar por fibrobroncoscopía para LBA y BTB.

El rendimiento diagnóstico de la $\mathrm{BTB}$ en la 


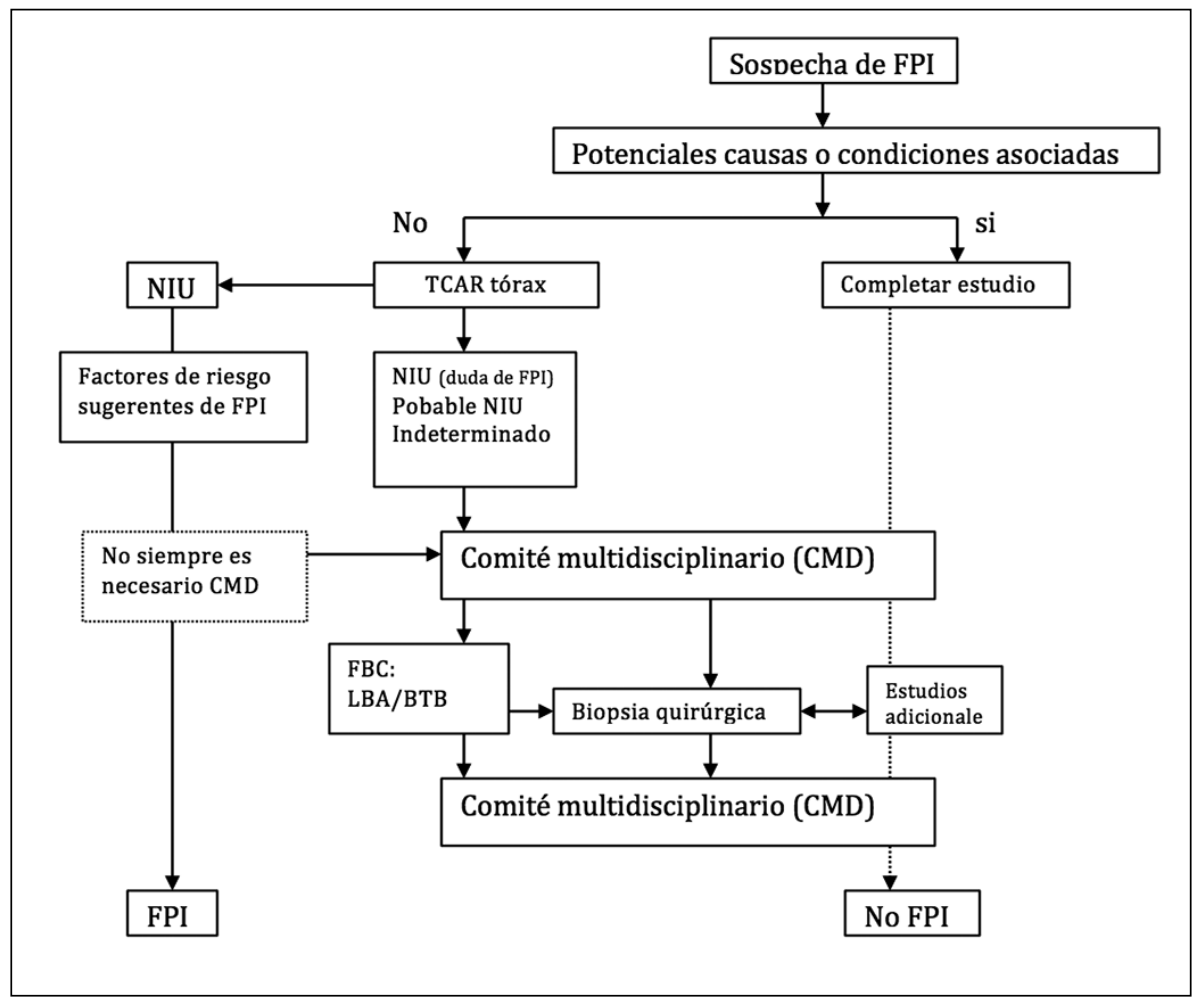

Figura 1. Algoritmo diagnóstico en pacientes con sospecha de FPI (según Raghu, et $\left.\mathrm{al}^{11}\right)$.
FPI es especialmente útil cuando hay sospecha de diagnósticos alternativos, como algunas NHS, la neumonía eosinofílica, la neumonía en organización o el cáncer (linfangitis carcinomatosa), la sarcoidosis o infección. En manos de expertos se logran obtener muestras adecuadas en $77,6 \%$ (IC95\% 74,6-80,3\%) y diagnósticos específicos en el 36\% (IC95\% 33,4-38,9\%) casos. El resto son inespecíficos o inclasificables ${ }^{1}$.

En los últimos años se ha desarrollado la técnica de criobiopsias pulmonares broncoscópicas ${ }^{12}$ para obtención de muestras más grandes que las obtenidas en las transbronquiales clásicas. El reciente estudio COLDICE ${ }^{13}$, comparó el uso de criobiopsia $v s$ biopsia por videotoracoscopía ambos asociadas a la evalaución por CMD. La adición de información de biopsia se consideró útil si cambiaba el diagnóstico de baja a alta confianza o definitivo, o proporcionaba un diagnóstico no anticipado. En $74 \%$ de los casos la criobiopsia aportó información y cambio de diagnóstico o terapia en el CMD y en $77 \%$ de la biopsias quirúrgicas. Este estudio mostró un alto nivel de concordancia entre ambos procedimientos, siempre apoyado con un CMD. Por lo que podría ser una opción a considerar, aunque aun con limitaciones en la implementación, el costo y la disponibilidad en centros.
Cuando la combinación de la información clínica, serológica y radiológica no permite llegar a un diagnóstico de confianza, se debe considerar la realización de una biopsia pulmonar quirúrgica por videotoracoscopía, si se piensa que su resultado va a influir en la decisión terapéutica.

Siempre es recomendable tomar muestras de múltiples lóbulos, evitando las áreas más severamente afectadas con fibrosis avanzada, probablemente no diagnóstica. La biopsia por videotoracoscopía debe evitarse en pacientes muy frágiles, con múltiples comorbilidades, con necesidad de oxígeno en reposo, con hipertensión pulmonar severa y $\mathrm{DL}_{\mathrm{CO}}$ severamente reducida $(<35 \%)^{14}$. Se prefiere la técnica toracoscópica video-asistida en 2 o 3 lóbulos. El rendimiento para diagnóstico específico es de 88,2\% (IC95\% 86,9-89,4\%). La Tabla 1 compara la frecuencia de complicaciones de las distintas formas de obtener histología utilizadas actualmente en paciente con EPI.

La combinación de la información emanada del análisis del patrón tomográfico y el histopatológico permite cruzarlos para decidir diagnósticos finales de FPI o no FPI (Tabla 2).

Alrededor de un $10 \%$ de casos que son clasificados como inclasificables. Esto se refiere a aquellos casos que el CMD encuentra conflictos insolubles entre los hallazgos clínicos, radioló- 
Tabla 1. Comparación de complicaciones por procedimientos para obtener histopatología*

\begin{tabular}{|c|c|c|c|}
\hline Complicaciones & Biopsia transbronquial & Criobiopsia & Biopsia quirúrgica \\
\hline Infección respiratoria & & $0,7 \%(\mathrm{IC} 95 \% 0,2-2 \%)$ & $6,5 \%(\mathrm{IC} 95 \% 5-9 \%)$ \\
\hline Exacerbación & & $1,2 \%$ (IC95\% 0,2-7\%) & $6,1 \%$ (IC95\% 5-7\%) \\
\hline Neumotórax & $10,2 \%($ IC95\% 4-22\%) & $16,5 \%$ (IC95\% 14-20\%) & \\
\hline Fuga aérea prolongada & $6,1 \%(\mathrm{IC} 95 \% 2-16,5 \%)$ & $13,4 \%$ (IC95\% 10-17\%) & $5,9 \%(\mathrm{IC} 95 \% 5-7 \%)$ \\
\hline Cualquier sangrado & & $5,2 \%(\mathrm{IC} 95 \% 4-7 \%)$ & $0,8 \%(\mathrm{IC} 95 \% 0,4-2 \%)$ \\
\hline Sangrado significativo & & $0,7 \%($ IC $95 \% 0,3-2 \%)$ & $0,2 \%$ (IC95\% 0,04-1,2\%) \\
\hline Dolor neuropático & & & $4,5 \%(\mathrm{IC} 95 \% 2-12,5 \%)$ \\
\hline Curación tardía de herida & & & $3,3 \%(\mathrm{IC} 95 \% 2-5,4 \%)$ \\
\hline Mortalidad general & $0 \%$ & $2,7 \%(\mathrm{IC} 95 \% 2-4 \%)$ & $3,5 \%(\mathrm{IC} 95 \% 3-4 \%)$ \\
\hline Mortalidad relacionada al procedimiento & & $0,2 \%(\mathrm{IC} 95 \% 0,04-1,3 \%)$ & $1,7 \%(\mathrm{IC} 95 \% 0,8-3,5 \%)$ \\
\hline
\end{tabular}

*Se presentan valores en porcentajes promedios de estudios agrupados, según Raghu et al ${ }^{11}$.

Tabla 2. Diagnóstico de Fibrosis Pulmonar Idiopática basado en patrones tomográficos e histológicos ${ }^{11}$

\begin{tabular}{|c|c|c|c|c|c|}
\hline \multirow{2}{*}{\multicolumn{2}{|c|}{$\begin{array}{l}\text { Sospecha de FPI } \\
\text { NIU }\end{array}$}} & \multicolumn{4}{|c|}{ Patrón histopatológico } \\
\hline & & Probable NIU & Indeterminado & Alternativo & \\
\hline \multirow{4}{*}{$\begin{array}{l}\text { Patrón } \\
\text { tomográfico }\end{array}$} & NIU & FPI & FPI & FPI & Dg No FPI \\
\hline & Probable NIU & FPI & FPI & FPI (probable)* & Dg No FPI \\
\hline & Indeterminado & FPI & FPI (probable)* & $\begin{array}{c}\text { Indeterminado } \\
\text { para } \mathrm{PFI} * *\end{array}$ & Dg No FPI \\
\hline & Alternativo & $\begin{array}{l}\text { FPI (probable)*/ } \\
\text { Dg No FPI }\end{array}$ & Dg No FPI & Dg No FPI & Dg No FPI \\
\hline
\end{tabular}

*FPI es diagnóstico $(\mathrm{Dg})$ probable cuando está presente cualquiera de los siguientes:

- Bronquiectasias/bronquiolectiasias por tracción moderadas a severas (leves en 4 o más lóbulos incluyendo la língula como lóbulo; o moderadas a severas en 2 o más lóbulos) en un hombre $>50$ años o en una mujer $>60$ años.

- Reticulación extensa $(>30 \%)$ en TC tórax $>70$ años.

- Aumento de neutrófilos y/o ausencia de linfocitosis en LBA.

- El CMD llega a un diagnóstico de confianza de FPI.

**En el caso indeterminado no es posible hacer un diagnóstico porque no se puede llegar a diagnóstico sin una biopsia adecuada y cuando la hay, puede ser reclasificada como un diagnóstico más específico, después de CMD y/o consultas adicionales.

gicos o histopatológicos o cuando por diversas razones no pueden realizarse procedimientos diagnósticos. El seguimiento de estos casos ha demostrado que tienen mejor sobrevida que los de NIU/FPI (HR 0,62, p = 0,04) y los factores independientes de sobrevida son la $\mathrm{DL}_{\mathrm{CO}}$ $(p=0,001)$ y el puntaje de fibrosis en la radiología $(\mathrm{p}=0,02)^{15}$.

Es importante recalcar que aunque existe una gran cantidad de literatura que informa sobre los beneficios del CMD para el diagnóstico de las EPI, y esto ha sido también nuestra experiencia de años, queda un debate considerable sobre qué constituye la mejor práctica o si existe un único modelo ideal de CMD. Las recomendaciones de la guía dejan, por lo tanto, sin responder muchas preguntas relacionadas con la práctica de la DMD.

Es de destacar que, a pesar de muchos años de investigación que destaca los beneficios del diagnóstico multidisciplinario de la EPI, la pregunta fundamental de cómo se valida la precisión del diagnóstico multidisciplinario no ha recibido respuesta. Por ahora en nuestra opinión el CMD debe ser el pilar fundamental en la toma de decisiones clínicas en los pacientes con EPI, sobre todo cuando estos plantean un reto diagnóstico individual. 


\section{Bibliografía}

1.- AMERICAN THORACIC SOCIETY. Idiopathic pulmonary fibrosis: diagnosis and treatment. International consensus statement. American Thoracic Society (ATS), and the European Respiratory Society (ERS). Am J Respir Crit Care Med 2000; 161 (2 Pt 1): 646-64.

2.- NICHOLSON AG, ADDIS BJ, BHARUCHA H, CLELLAND CA, CORRIN B, GIBBS AR, et al. Inter-observer variation between pathologists in diffuse parenchymal lung disease. Thorax 2004; 59: 500-5.

3.- FLAHERTY KR, TRAVIS WD, COLBY TV, TOEWS GB, KAZEROONI EA, GROSS BH, et al. Histopathologic variability in usual and nonspecific interstitial pneumonias. Am J Respir Crit Care Med 2001; 164: 1722-7.

4.- AZIZ ZA, WELLS AU, HANSELL DM, BAIN GA, COPLEY SJ, DESAI SR, et al. HRCT diagnosis of diffuse parenchymal lung disease: inter-observer variation. Thorax 2004; 59: 506-11.

5.- LEVI Y, ISRAELI-SHANI L, KUCHUK M, EPSTEIN SHOCHET G, KOSLOW M, SHITRIT D. Rheumatological assessment is important for Interstitial Lung Disease Diagnosis. J Rheumatol 2018; 45: 150914.

6.- WALSH SLF, WELLS AU, DESAI SR, POLETTI V, PICIUCCHI S, DUBINI A, et al. Multicentre evaluation of multidisciplinary team meeting agreement on diagnosis in diffuse parenchymal lung disease: a casecohort study. Lancet Respir Med 2016; 4: 557-65.

7.- WALSH SLF. Multidisciplinary evaluation of interstitial lung diseases: current insights. Eur Respir Rev 2017; 26: 170002.

8.- LEDERER DJ, MARTÍNEZ FJ. Idiopathic Pulmonary Fibrosis. N Engl J Med 2018; 378: 1811-23.

9.- BROWNELL R, MOUA T, HENRY TS, ELICKER
BM, WHITE D, VITTINGHOFF E, et al. The use of pretest probability increases the value of high-resolution CT in diagnosing usual interstitial pneumonia. Thorax 2017; 72: 424-9.

10.- LYNCH DA, SVERZELLATI N, TRAVIS WD, BROWN KK, COLBY TV, GALVIN JG, et al. Diagnostic criteria for idiopathic pulmonary fibrosis: a Fleischner Society White Paper. Lancet Respir Med 2018; 6: 138-53.

11.- RAGHU G, REMY-JARDIN M, MYERS JL, RICHELDI L, RYERSON CJ, LEDERER DJ, et al. Diagnosis of idiopathic pulmonary fibrosis: an official ATS/ERS/JRS/ALAT clinical practice guideline. Am J Respir Crit Care Med 2018; 198: e44-e68.

12.- TOMASSETTI S, WELLS AU, COSTABEL U,CAVAZZA A, COLBY TV, ROSSI G, et al. Bronchoscopic lung cryobiopsy increases diagnostic confidence in the multidisciplinary diagnosis of idiopathic pulmonary fibrosis. Am J Respir Crit Care Med 2016; 193: 745-52.

13.- TROY LK, GRAINGE C, CORTE TJ, WILLIAMSON JP, VALLELY MP, COOPER WA. Diagnostic accuracyof transbronchial for interstitial lung disease diagnosis (COLDICE): a prospective, comparative study. Lancet Respir Med 2019; (published online Sept 29) https://doi.org/10.1016/S22132600(19)30342-X.

14.- HUTCHINSON JP, FOGARTY AW, MCKEEVER TM, HUBBARD RB. In-hospital mortality after surgical lung biopsy for interstitial lung disease in the United States: 2000 to 2011. Am J Respir Crit Care Med 2016; 193: 1161-7.

15.- RYERSON C, URBANI T, RICHELDI L, MOONEY JJ, LEE LS, JONES KD, et al. Prevalence and prognosis of unclassifiable interstitial lung disease. Eur Respir J 2013; 42: 750-7.

Correspondencia a:

Dr. Felipe Reyes C.

Email: freyes.cartes@gmail.com 